\title{
Recursive Estimation and Identification of Wireless Ad Hoc Channels from Measurements
}

\author{
M. Olama Y. Li S. Djouadi \\ University of Tennessee \\ 1508 Middle Drive \\ Knoxville, TN 37919, USA \\ Tel: 0018659745447
}

\{molama, yli24, mdjouadi\}@utk.edu

\author{
T. Goodspeed T. Kuruganti \\ Oak Ridge National Laboratory \\ 1 Bethel Valley Road, MS-6085 \\ Oak Ridge, TN 37831 \\ Tel: 0018652412874
}

\{goodspeedtm, kurugantipv\}@ornl.gov

\begin{abstract}
This paper is concerned with a time varying wireless ad hoc channel modeling, its parameter estimation and system identification from received signal measurement data. The channel model is represented in state space form, while the expectation maximization algorithm and Kalman filtering are used in the channel parameter and state estimation, respectively. The proposed algorithm is recursive, and therefore the inphase and quadrature components of the ad hoc channel and its parameters are estimated online from received signal measurements. The proposed algorithm is tested using measurement data collected from moving wireless sensor nodes, and the results are presented.
\end{abstract}

\section{Keywords}

Ad hoc channel, expectation maximization algorithm, Kalman filter, stochastic state space representation.

\section{INTRODUCTION}

Time varying (TV) wireless ad hoc channel models capture both the space and time variations of wireless systems, such as wireless sensor networks. These variations are due to the relative mobility of the receiver, transmitter and/or scatterers [1], [2]. This paper is concerned with the development of TV wireless channel models based on system identification algorithms to extract various ad hoc channel parameters using received signal measurement data.

The majority of research papers in this field such as in [3], [4] use time-invariant (static) models for wireless channels. In timeinvariant models, channel parameters are random but do not depend on time, and remain constant throughout the observation and estimation phase. This contrasts with TV models, where the channel dynamics become TV stochastic processes [1], [2].

In [1] and [2], the TV channel parameters are estimated from approximating the Doppler power spectral density (DPSD) of the wireless ad hoc channel. However, in reality one can not have

Permission to make digital or hard copies of all or part of this work for personal or classroom use is granted without fee provided that copies are not made or distributed for profit or commercial advantage and that copies bear this notice and the full citation on the first page. To copy otherwise, to republish, to post on servers or to redistribute to lists, requires prior specific permission and/or a fee. WICON 2007, October 22-24, Austin, USA

Copyright (C) 2007 978-963-9799-12-7

DOI 10.4108/pwsn.2007.2264 access to the TV DPSD at all times during the estimation process. We propose to estimate channel parameters as well as the inphase and quadrature components directly from the received signal measurements, which are usually available or easy to obtain in any wireless ad hoc or sensor network. The expectation maximization (EM) algorithm [6] and Kalman filtering [7] are employed in the estimation process. The proposed algorithm is recursive, and therefore can be implemented in real time. The proposed models and estimation algorithm are tested using measurement data collected from moving Crossbow's TelosB wireless sensor nodes. The experimental setup and results are presented.

These models are important in the development of a practical channel simulator that replicates wireless channel characteristics, and produces outputs that vary in a similar manner to the variations encountered in a real-world channel environment.

The paper is organized as follows. In Section 2, the TV wireless ad hoc channel mathematical model is introduced. In Section 3, the EM algorithm together with the Kalman filter, to estimate the channel parameters as well as the inphase and quadrature components from received signal measurements, is developed. In Section 4, experimental setup and numerical results are presented. Finally, Section 5 provides the conclusion.

\section{MATHEMATICAL MODELS FOR TV WIRELESS AD HOC CHANNELS}

The general TV model of a wireless channel is typically represented by the band-pass impulse response [5]:

$H(t ; \tau)=\sum_{j=1}^{J(t)}\left(I_{j}(t, \tau) \cos \left(\omega_{c} t\right)-Q_{j}(t, \tau) \sin \left(\omega_{c} t\right)\right) \delta\left(\tau-\tau_{j}(t)\right)(1)$

where $H(t ; \tau)$ is the band-pass response of the channel at time $t$, due to an impulse applied at time $t-\tau, J(t)$ is the random number of multipath components, $\omega_{c}$ is the carrier frequency, and the set $\left\{I_{j}(t, \tau), Q_{j}(t, \tau), \tau_{j}(t)\right\}_{j=1}^{J(t)}$ describes the random TV inphase component, quadrature component, and arrival time of the different paths, respectively. Let $s_{l}(t)$ be the low pass equivalent representation of the transmitted signal, then the band pass representation of the received signal is given by: 


$$
\begin{gathered}
y(t)=\sum_{j=1}^{J(t)}\left(I_{j}(t, \tau) \cos \left(\omega_{c} t\right)-Q_{j}(t, \tau) \sin \left(\omega_{c} t\right)\right) s_{l}\left(t-\tau_{j}(t)\right) \\
+v_{I}(t) \cos \left(\omega_{c} t\right)-v_{Q}(t) \sin \left(\omega_{c} t\right)
\end{gathered}
$$

where $\left\{v_{I}(t)\right\}_{t \geq 0}$ and $\left\{v_{Q}(t)\right\}_{t \geq 0}$ are two independent and identically distributed (iid) white Gaussian noise processes, with density $\mathcal{N}\left(0 ; \sigma_{v}^{2}\right)$.

It is shown in [1] that the DPSD of a wireless ad hoc channel, denoted by $S(s)$, can be approximated by an even, stable, rational, and factorizable transfer function, $\tilde{S}(s)=H(s) H(-s)$, where $H(s)$ is:

$$
H(s)=\frac{b_{n-1}^{\prime} s^{n-1}+\ldots+b_{1}^{\prime} s+b_{0}^{\prime}}{s^{n}+a_{n-1}^{\prime} s^{n-1}+\ldots+a_{1}^{\prime} s+a_{0}^{\prime}}
$$

Consequently, the inphase and quadrature components can be realized using the following stochastic observable canonical form (OCC) state space representation:

$$
\begin{aligned}
& d X_{I, j}(t)=A_{I} X_{I, j}(t) d t+B_{I} d W_{j}^{I}(t) \\
& I_{j}(t)=C_{I} X_{I, j}(t)+f_{j}^{I}(t) \\
& d X_{Q, j}(t)=A_{Q} X_{Q, j}(t) d t+B_{Q} d W_{j}^{Q}(t) \\
& Q_{j}(t)=C_{Q} X_{Q, j}(t)+f_{j}^{Q}(t)
\end{aligned}
$$

where

$$
\begin{aligned}
& X_{I, j}(t)=\left[X_{I, j}^{1}(t), X_{I, j}^{2}(t), \ldots, X_{I, j}^{n}(t)\right]^{T}, \\
& X_{Q, j}(t)=\left[X_{Q, j}^{1}(t), X_{Q, j}^{2}(t), \ldots, X_{Q, j}^{n}(t)\right]^{T}, \\
& A_{I}=A_{Q}=\left[\begin{array}{ccccc}
0 & 1 & 0 & \cdots & 0 \\
0 & 0 & 1 & \cdots & 0 \\
\vdots & \vdots & \vdots & \ddots & \vdots \\
0 & 0 & 0 & \cdots & 1 \\
-a_{0}^{\prime} & -a_{1}^{\prime} & -a_{2}^{\prime} & -a_{n-1}^{\prime}
\end{array}\right] \\
& B_{I}=B_{Q}=\left[\begin{array}{c}
b_{n-1}^{\prime} \\
\vdots \\
\vdots \\
b_{1}^{\prime} \\
b_{0}^{\prime}
\end{array}\right], C_{I}=C_{Q}=\left[\begin{array}{llll}
1 & 0 & \cdots & 0
\end{array}\right]
\end{aligned}
$$

$\left\{W_{j}^{I}(t)\right\}_{t \geq 0}$ and $\left\{W_{j}^{Q}(t)\right\}_{t \geq 0}$ are two independent standard Brownian motions, which correspond to the inphase and quadrature components of the $j$ th path respectively, $f_{j}^{I}(t)$ and $f_{j}^{Q}(t)$ are arbitrary functions representing the line-of-sight (LOS) of the inphase and quadrature components for the $j$ th path respectively, and $T$ is vector or matrix transpose. Without loss of generality, we consider the case of flat fading, in which the fading channel has purely a multiplicative effect on the signal and the multipath components are not resolvable. Thus, it can be considered as a single path [5]. We also consider the non-line-ofsight (NLOS) case, i.e., $f_{j}^{I}(t)=f_{j}^{Q}(t)=0$, which represents an environment with large obstructions.

The channel model in (4) assumes that mobile nodes move at constant velocities. But in reality, the propagation environment varies continuously due to movement of the nodes at variable speeds and directions, the angle of arrival of the wave upon the receiver can vary continuously, and objects or scatters move in between the transmitter and the receiver resulting in appearance or disappearance of existing paths from one instant to the next. As a consequence of these variations, the statistical characteristics of the received signal vary continuously, giving rise to a DPSD which varies from one observation instant to the next. Therefore, the channel parameters $A_{I}, A_{Q}, B_{I}$, and $B_{Q}$ in (4) are time varying and represented as $A_{I}(t), A_{Q}(t), B_{I}(t)$, and $B_{Q}(t)$. Solution of the time varying version of the state space model in (4) is given by:

$$
X_{L}(t)=\Phi_{L}\left(t, t_{0}\right) X_{L}\left(t_{0}\right)+\int_{t_{0}}^{t} \Phi_{L}(t, u) B_{L}(u) d W_{L}(u)
$$

where $L=I$ or $Q, \Phi_{L}\left(t, t_{0}\right)$ is the fundamental matrix, and $\dot{\Phi}_{L}\left(t, t_{0}\right)=A_{L}(t) \Phi_{L}\left(t, t_{0}\right)$. Therefore, the mean of $X_{L}(t)$ is:

$$
E\left[X_{L}(t)\right]=\Phi_{L}\left(t, t_{0}\right) E\left[X_{L}\left(t_{0}\right)\right]
$$

and the covariance matrix of $X_{L}(t)$ is:

$$
\begin{aligned}
\Sigma_{L}(t)= & \Phi_{L}\left(t, t_{0}\right) \operatorname{Var}\left[X_{L}\left(t_{0}\right)\right] \Phi_{L}^{T}\left(t, t_{0}\right) \\
& +\int_{t_{0}}^{t} \Phi_{L}(t, u) B_{L}(u) B_{L}^{T}(u) \Phi_{L}^{T}\left(t, t_{0}\right) d u
\end{aligned}
$$

For the time invariant case, $A_{L}(t)=A_{L}$ and $B_{L}(t)=B_{L}$, then (6), (7), and (8) simplify to:

$$
\begin{gathered}
X_{L}(t)=e^{A_{L}\left(t-t_{0}\right)} X_{L}\left(t_{0}\right)+\int_{t_{0}}^{t} e^{A_{L}(t-u)} B_{L} d W_{L}(u) \\
E\left[X_{L}(t)\right]=e^{A_{L}\left(t-t_{0}\right)} E\left[X_{L}\left(t_{0}\right)\right] \\
\Sigma_{L}(t)=e^{A_{L}\left(t-t_{0}\right)} \operatorname{Var}\left[X_{L}\left(t_{0}\right)\right] e^{A_{L}^{T}\left(t-t_{0}\right)} \\
+\int_{t_{0}}^{t} e^{A_{L}(t-u)} B_{L} B_{L}^{T} e^{A_{L}^{T}(t-u)} d u
\end{gathered}
$$


It can be seen in (7) and (8) that the mean and variance of the inphase and quadrature components are functions of time. Thus the statistics of the inphase and quadrature components, and therefore the statistics of the ad hoc channel, are times varying. Indeed, these stochastic state space models reflect the TV characteristics of the ad hoc channel.

Similarly, following the state space representation in (4), the ad hoc channel can be represented using general stochastic state space representation of the form:

$$
\begin{aligned}
& d X(t)=A(t) X(t) d t+B(t) d W(t) \\
& y(t)=C(t) X(t)+D(t) v(t)
\end{aligned}
$$

where

$$
\begin{aligned}
& X(t)=\left[\begin{array}{ll}
X_{I}(t) & X_{Q}(t)
\end{array}\right]^{T}, \\
& A(t)=\left[\begin{array}{cc}
A_{I}(t) & 0 \\
0 & A_{Q}(t)
\end{array}\right], B(t)=\left[\begin{array}{cc}
B_{I}(t) & 0 \\
0 & B_{Q}(t)
\end{array}\right], \\
& C(t)=\left[\begin{array}{ll}
\cos \left(\omega_{c} t\right) C_{I} & -\sin \left(\omega_{c} t\right) C_{Q}
\end{array}\right], \\
& D(t)=\left[\begin{array}{ll}
\cos \left(\omega_{c} t\right) & -\sin \left(\omega_{c} t\right)
\end{array}\right] \\
& d W(t)=\left[\begin{array}{ll}
d W^{I}(t) & d W^{Q}(t)
\end{array}\right]^{T} \\
& v(t)=\left[\begin{array}{ll}
v_{I}(t) & v_{Q}(t)
\end{array}\right]^{T}
\end{aligned}
$$

In this case, $y(t)$ represents the received signal measurement, $X(t)$ is the state variable of the inphase and quadrature components, and $v(t)$ is the measurement noise, which is assumed to be Gaussian.

In [1] and [2], the channel parameters $\left\{a_{n-1}^{\prime}, \ldots, a_{0}^{\prime}, b_{n-1}^{\prime}, \ldots, b_{0}^{\prime}\right\}$ are obtained from approximating the DPSD. However, in reality one can not have access to the DPSD at all times during the estimation process. In this paper, the channel parameters as well as the inphase and quadrature components are estimated directly from received signal measurements, which are usually available or easy to obtain in any wireless network. The EM algorithm and Kalman filtering are employed in the channel parameter and state estimation, respectively. These algorithms are introduced in the next section.

\section{WIRELESS AD HOC CHANNEL ESTIMATION VIA THE EM ALGORITHM AND KALMAN FILTERING}

This section describes the procedure employed to estimate the channel model parameters and states associated with the state space model in (10), using the EM algorithm [6] together with Kalman filtering [7]. However, for simplicity we consider the discrete-time version of (10) given by:

$$
\begin{aligned}
& x_{t+1}=A_{t} x_{t}+B_{t} w_{t} \\
& y_{t}=C_{t} x_{t}+D_{t} v_{t}
\end{aligned}
$$

where $x_{t} \in \mathfrak{R}^{n}$ is a state vector, $y_{t} \in \mathfrak{R}^{d}$ is a measurement vector, $w_{t} \in \mathfrak{R}^{m}$ is a state noise, and $v_{t} \in \mathfrak{R}^{d}$ is a measurement noise. The noise processes $w_{t}$ and $v_{t}$ are assumed to be independent zero mean and unit variance Gaussian processes.

The unknown system parameters $\theta_{t}=\left\{A_{t}, B_{t}, C_{t}, D_{t}\right\}$ as well as the system states $x_{t}$ are estimated through the received signal measurement data, $Y_{N}=\left\{y_{1}, y_{2}, \ldots, y_{N}\right\}$. The methodology employed is recursive and based on the EM algorithm together with the Kalman filter. The Kalman filter is introduced next.

\subsection{Channel State Estimation: The Kalman Filter}

The Kalman filter estimates the channel states $x_{t}$ for given system parameter $\theta_{t}$ and measurements $Y_{t}$. It is described by the following equations [7]:

$$
\begin{aligned}
& \hat{x}_{t \mid t}=A_{t} \hat{x}_{t-1 \mid t-1}+P_{t \mid t} C_{t}^{T} D_{t}^{-2}\left(y_{t}-C_{t} A_{t} \hat{x}_{t-1 \mid t-1}\right) \\
& \hat{x}_{t \mid t-1}=A_{t} \hat{x}_{t-1 \mid t-1}, \hat{x}_{0 \mid 0}=m_{0}
\end{aligned}
$$

where $t=0,1,2, \ldots, N$, and $P_{t \mid t}$ is given by:

$$
\begin{aligned}
& \bar{P}_{t \mid t}^{-1}=P_{t-1 \mid t-1}^{-1}+A_{t}^{T} B_{t}^{-2} A_{t} \\
& P_{t \mid t}^{-1}=C_{t}^{T} D_{t}^{-2} C_{t}+B_{t}^{-2}-B_{t}^{-2} \bar{P}_{t \mid t} A_{t}^{T} B_{t}^{-2} \\
& P_{t \mid t-1}=A_{t} P_{t-1 \mid t-1} A_{t}^{T}+B_{t}^{2}
\end{aligned}
$$

The channel parameters $\theta_{t}=\left\{A_{t}, B_{t}, C_{t}, D_{t}\right\}$ are estimated using the EM algorithm which is introduced next.

\subsection{Channel Parameter Estimation: The EM Algorithm}

The EM algorithm uses a bank of Kalman filters to yield a maximum likelihood (ML) parameter estimate of the Gaussian state space model. Let $\theta_{t}=\left\{A_{t}, B_{t}, C_{t}, D_{t}\right\}$ denotes the system parameters in (12), $P_{0}$ denotes a fixed probability measure; and $\left\{P_{\theta_{t}} ; \theta_{t} \in \Theta\right\}$ denotes a family of probability measures induced by the system parameters $\theta_{t}$. If the original model is a white noise sequence, then $\left\{P_{\theta_{t}} ; \theta_{t} \in \Theta\right\}$ is absolutely continuous with respect to $P_{0}$ [6]. Moreover, it can be shown that under $P_{0}$ we have:

$$
P_{0}:\left\{\begin{array}{c}
x_{t+1}=w_{t} \\
y_{t}=v_{t}
\end{array}\right.
$$


The EM algorithm is an iterative scheme for computing the ML estimate of the system parameters $\theta_{t}$, given the data $Y_{t}$. Specifically, each iteration of the EM algorithm consists of two steps: The expectation step and the maximization step.

The expectation step evaluates the conditional expectation of the log-likelihood function given the complete data, which is described by:

$$
\Lambda\left(\theta_{t}, \hat{\theta}_{t}\right)=E_{\theta_{l}}\left\{\log \frac{d P_{\theta_{t}}}{d P_{\hat{\theta}_{t}}} \mid Y_{t}\right\}
$$

where $\hat{\theta}_{t}$ denotes the estimated system parameters at time step $t$. The maximization step finds:

$$
\hat{\theta}_{t+1} \in \underset{\theta_{t} \in \Theta}{\arg \max } \Lambda\left(\theta_{t}, \hat{\theta}_{t}\right)
$$

The expectation and maximization steps are repeated until the sequence of model parameters converge to the real parameters.

For the system model in (12), the EM algorithm is described by the following equations [6]:

$$
\begin{aligned}
& \hat{A}_{t}=E\left(\sum_{k=1}^{t} x_{k} x_{k-1}^{T} \mid Y_{t}\right) \times\left[E\left(\sum_{k=1}^{t} x_{k} x_{k}^{T} \mid Y_{t}\right)\right]^{-1} \\
& \hat{B}_{t}^{2}=\frac{1}{t} E\left(\sum_{k=1}^{t}\left(\left(x_{k}-A_{k} x_{k-1}\right)\left(x_{k}-A_{k} x_{k-1}\right)^{T}\right) \mid Y_{t}\right) \\
& =\frac{1}{t} E\left(\sum_{k=1}^{t}\left(\left(x_{k} x_{k}^{T}\right)-A_{k}\left(x_{k} x_{k-1}^{T}\right)^{T}-\left(x_{k} x_{k-1}^{T}\right) A_{k}^{T}+A_{k}\left(x_{k-1} x_{k-1}^{T}\right) A_{k}^{T}\right) \mid Y_{t}\right) \\
& \hat{C}=E\left(\sum_{k=1}^{t} y_{k} x_{k}^{T} \mid Y_{t}\right) \times\left[E\left(\sum_{k=1}^{t} x_{k} x_{k}^{T} \mid Y_{t}\right)\right]^{-1} \\
& \hat{D}_{t}^{2}=\frac{1}{t} E\left(\sum_{k=1}^{t}\left(\left(y_{k}-C_{k} x_{k}\right)\left(y_{k}-C_{k} x_{k}\right)^{T}\right) \mid Y_{t}\right) \\
& \quad=\frac{1}{t} E\left(\sum_{k=1}^{t}\left(\left(y_{k} y_{k}^{T}\right)-\left(y_{k} x_{k}^{T}\right) C_{k}^{T}-C_{k}\left(y_{k} x_{k}^{T}\right)^{T}+C_{k}\left(x_{k} x_{k}^{T}\right) C_{k}^{T}\right) \mid Y_{t}\right)
\end{aligned}
$$

where $B_{t}^{2}=B_{t} B_{t}^{T}, D_{t}^{2}=D_{t} D_{t}^{T}$, and $E(\cdot)$ denotes the expectation operator. The system parameters $\left\{\hat{A}_{t}, \hat{B}_{t}^{2}, \hat{C}_{t}, \hat{D}_{t}^{2}\right\}$ can be computed from the following conditional expectations [6]:

$$
\begin{aligned}
& L_{t}^{(1)}=E\left\{\sum_{k=1}^{t} x_{k}^{T} Q x_{k} \mid Y_{t}\right\}, \\
& L_{t}^{(2)}=E\left\{\sum_{k=1}^{t} x_{k-1}^{T} Q x_{k-1} \mid Y_{t}\right\}, \\
& L_{t}^{(3)}=E\left\{\sum_{k=1}^{t}\left[x_{k}^{T} R x_{k-1}+x_{k-1}^{T} R^{T} x_{k}\right] \mid Y_{t}\right\}, \\
& L_{t}^{(4)}=E\left\{\sum_{k=1}^{t}\left[x_{k}^{T} S y_{k}+y_{k}^{T} S^{T} x_{k}\right] \mid Y_{t}\right\}
\end{aligned}
$$

$$
\begin{aligned}
& Q=\left\{\frac{e_{i} e_{j}^{T}+e_{j} e_{i}^{T}}{2}\right\}, R=\left\{\frac{e_{i} e_{j}^{T}}{2}\right\}, i, j=1,2, \ldots n \\
& S=\left\{\frac{e_{i} e_{l}^{T}}{2}\right\} ; i=1,2, \ldots n ; l=1,2, . . d
\end{aligned}
$$

in which $e_{i}$ is the unit vector in the Euclidean space; that is $e_{i}=1$ in the $i$ th position, and 0 elsewhere. For instance, consider the case $n=d=2$, then $E\left(\sum_{k=1}^{t} x_{k} x_{k-1}^{T} \mid Y_{t}\right)$ is described by:

$$
E\left(\sum_{k=1}^{t} x_{k} x_{k-1}^{T} \mid Y_{t}\right)=\left[\begin{array}{ll}
L_{t}^{(3)}\left(R_{11}\right) & L_{t}^{(3)}\left(R_{21}\right) \\
L_{t}^{(3)}\left(R_{12}\right) & L_{t}^{(3)}\left(R_{22}\right)
\end{array}\right]
$$

where $R_{i j}=\left\{e_{i} e_{j}^{T} / 2 ; i, j=1,2\right\}$. The other terms in (18) can be computed similarly.

The conditional expectations $\left\{L_{t}^{(1)}, L_{t}^{(2)}, L_{t}^{(3)}, L_{t}^{(4)}\right\}$ can be estimated from measurements $Y_{t}$ as follows:

1) Filter estimate of $L_{t}^{(1)}$ :

$$
\begin{aligned}
L_{t}^{(1)}= & E\left\{\sum_{k=1}^{t} x_{k}^{T} Q x_{k} \mid Y_{t}\right\}= \\
& -\frac{1}{2} \operatorname{Tr}\left(N_{t}^{(1)} P_{t \mid t}\right)-\frac{1}{2} \sum_{k=1}^{t} \operatorname{Tr}\left(N_{k-1}^{(1)} \bar{P}_{k \mid k}\right) \\
- & \frac{1}{2} \sum_{k=1}^{t}\left(\begin{array}{l}
-2 x_{k \mid k}^{T} P_{k \mid k}^{-1} r_{k}^{(1)}+2 x_{k \mid k-1}^{T} P_{k \mid k-1}^{-1} r_{k \mid k-1}^{(1)}-x_{k \mid k}^{T} N_{k}^{(1)} x_{k \mid k} \\
+x_{k \mid k-1}^{T} B_{k}^{-2} A_{k} \bar{P}_{k \mid k} N_{k-1}^{(1)} \bar{P}_{k \mid k} A_{k}^{T} B_{k}^{-2} x_{k \mid k-1}
\end{array}\right)
\end{aligned}
$$

where $\operatorname{Tr}(\cdot)$ denotes the matrix trace. In (22), $r_{k}^{(1)}$ and $N_{k}^{(1)}$ satisfy the following recursions:

$$
\begin{aligned}
& \left\{\begin{array}{c}
r_{k}^{(1)}=\left(A_{k}-P_{k \mid k} C_{k}^{T} D_{k}^{-2} C_{k} A_{k}\right) r_{k-1}^{(1)}+2 P_{k \mid k} Q x_{k \mid k-1} \\
\quad-P_{k \mid k} N_{k}^{(1)} P_{k \mid k} C_{k}^{T} D_{k}^{-2}\left(y_{k}-C_{k} x_{k \mid k-1}\right) \\
r_{k \mid k-1}^{(1)}=A_{k} r_{k}^{(1)} \\
r_{0}^{(1)}=0_{m \times 1}
\end{array}\right. \\
& \left\{\begin{array}{c}
N_{k}^{(1)}=B_{k}^{-2} A_{k} \bar{P}_{k \mid k} N_{k-1}^{(1)} \bar{P}_{k \mid k} A_{k}^{T} B_{k}^{-2}-2 Q \\
N_{0}^{(1)}=0_{m \times m}
\end{array}\right.
\end{aligned}
$$

2) Filter estimate of $L_{t}^{(2)}$ :

$$
\begin{aligned}
& L_{t}^{(2)}=E\left\{\sum_{k=1}^{t} x_{k-1}^{T} Q x_{k-1} \mid Y_{t}\right\}= \\
& E_{\theta}\left\{x_{0}^{T} Q x_{0} \mid Y_{t}\right\}+E_{\theta}\left\{\sum_{k=1}^{t} x_{k}^{T} Q x_{k} \mid Y_{t}\right\}-E_{\theta}\left\{x_{t}^{T} Q x_{t} \mid Y_{t}\right\}
\end{aligned}
$$

where $Q, R$ and $S$ are given by:

Therefore, $L_{t}^{(2)}$ can be obtained from $L_{t}^{(1)}$. 
3) Filter estimate of $L_{t}^{(3)}$ :

$$
\begin{aligned}
L_{t}^{(3)}= & E\left\{\sum_{k=1}^{t}\left(x_{k}^{T} R x_{k-1}+x_{k-1}^{T} R^{T} x_{k}\right) \mid Y_{t}\right\}= \\
& -\frac{1}{2} \operatorname{Tr}\left(N_{t}^{(3)} P_{t \mid t}\right)-\frac{1}{2} \sum_{k=1}^{t} \operatorname{Tr}\left(N_{k-1}^{(3)} \bar{P}_{k \mid k}\right) \\
- & \frac{1}{2} \sum_{k=1}^{t}\left(\begin{array}{l}
-2 x_{k \mid k}^{T} P_{k \mid k}^{-1} r_{k}^{(3)}+2 x_{k \mid k-1}^{T} P_{k \mid k-1}^{-1} r_{k \mid k-1}^{(3)}-x_{k \mid k}^{T} N_{k}^{(3)} x_{k \mid k} \\
+x_{k \mid k-1}^{T} B_{k}^{-2} A_{k} \bar{P}_{k \mid k} N_{k-1}^{(3)} \bar{P}_{k \mid k} A_{k}^{T} B_{k}^{-2} x_{k \mid k-1}
\end{array}\right)
\end{aligned}
$$

In this case, $r_{k}^{(3)}$ and $N_{k}^{(3)}$ satisfy the following recursions:

$$
\begin{aligned}
& \left\{\begin{aligned}
r_{k}^{(3)}= & \left(A_{k}-P_{k \mid k} C_{k}^{T} D_{k}^{-2} C_{k} A_{k}\right) r_{k-1}^{(3)}-P_{k \mid k} N_{k}^{(3)} P_{k \mid k} C_{k}^{T} D_{k}^{-2} \\
& \left(y_{k}-C_{k} x_{k \mid k-1}\right)+\left(2 P_{k \mid k} R+2 P_{k \mid k} B_{k}^{-2} A_{k} \bar{P}_{k \mid k} R^{T} A_{k}\right) x_{k-1 \mid k-1} \\
r_{k \mid k-1}^{(3)}= & A_{k} r_{k}^{(3)} \\
r_{0}^{(3)}= & 0_{m \times 1}
\end{aligned}\right. \\
& \left\{\begin{array}{c}
N_{k}^{(3)}=B_{k}^{-2} A_{k} \bar{P}_{k \mid k} N_{k-1}^{(3)} \bar{P}_{k \mid k} A_{k}^{T} B_{k}^{-2}-2 R \bar{P}_{k \mid k} A_{k}^{T} B_{k}^{-2} \\
-2 B_{k}^{-2} A_{k} \bar{P}_{k \mid k} R^{T} \\
N_{0}^{(3)}=0_{m \times m}
\end{array}\right.
\end{aligned}
$$

4) Filter estimate of $L_{t}^{(4)}$ :

$$
\begin{aligned}
& L_{t}^{(4)}=E\left\{\sum_{k=1}^{t}\left(x_{k}^{T} S y_{k}+y_{k}^{T} S^{T} x_{k}\right) \mid Y_{t}\right\} \\
& =\sum_{k=1}^{t}\left(x_{k \mid k}^{T} P_{k \mid k}^{-1} r_{k}^{(4)}-x_{k \mid k-1}^{T} P_{k \mid k-1}^{-1} r_{k \mid k-1}^{(4)}\right)
\end{aligned}
$$

where $r_{k}^{(4)}$ satisfy the following recursions:

$$
\left\{\begin{array}{l}
r_{k}^{(4)}=\left(A_{k}-P_{k \mid k} C_{k}^{T} D_{k}^{-2} C_{k} A_{k}\right) r_{k-1}^{(4)}+2 P_{k \mid k} S y_{k} \\
r_{k \mid k-1}^{(4)}=A_{k} r_{k}^{(4)} \\
r_{0}^{(4)}=0_{m \times 1}
\end{array}\right.
$$

Using the filters for $L_{t}^{(i)}(i=1,2,3,4)$ and the Kalman filter described earlier, the system parameters $\theta_{t}=\left\{A_{t}, B_{t}, C_{t}, D_{t}\right\}$ can be estimated through the EM algorithm described in (18). Experimental results that show the applicability of the above algorithm in estimating the channel parameters as well as the inphase and quadrature components from measurements are discussed next.

\section{EXPERIMENTAL RESULTS}

In this section, we perform experiments to measure the received signal power of moving sensors in wireless sensor network platform, and then the developed EM algorithm together with Kalman filtering is used to estimate the ad hoc channel parameters as well as the inphase and quadrature components from received signal measurements. The wireless sensors used in our experiment are Crossbow's TelosB sensor nodes. A single
TelosB sensor node is shown in Figure 1. It has the following specifications: IEEE 802.15.4 Compliant, data rate is $250 \mathrm{kbps}$, carrier frequency is $2.4 \mathrm{GHz}$, and has USB interface. This sensor is implemented with a Chipcon CC2420 RF transceiver chip which provides a built-in received signal strength indicator (RSSI). This indicator is averaged over 8 symbol periods (128 micro second). The RSSI has a dynamic range of $100 \mathrm{~dB}$ and is accurate to $+/-6 \mathrm{~dB}[8]$.

Experimental setup consists of two moving transceivers (sensors 1 and 2) and one passive receiver (sensor 3) connected to a workstation as shown in Figure 2. At each time step, sensors 1 and 2 each broadcast a packet containing a source address and the

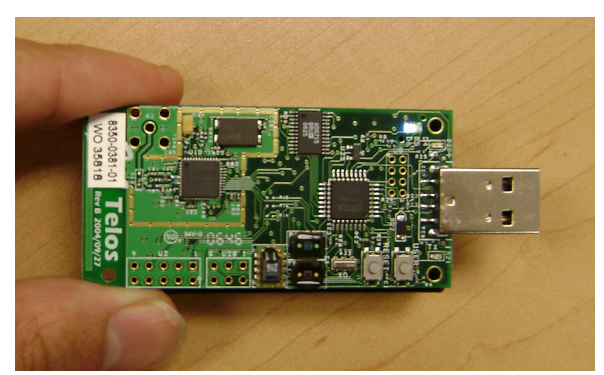

Figure 1. Crossbow TelosB sensor node.

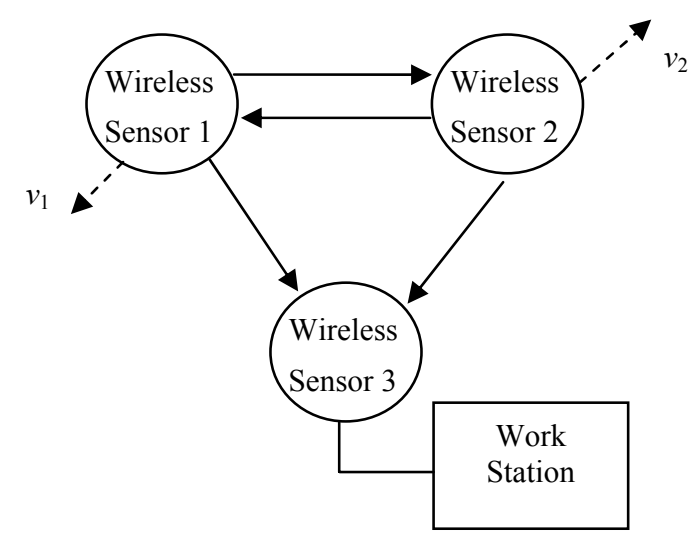

Figure 2. Experimental setup: Two moving transceivers (sensors 1 and 2 ) and one fixed receiver (sensor 3 ).

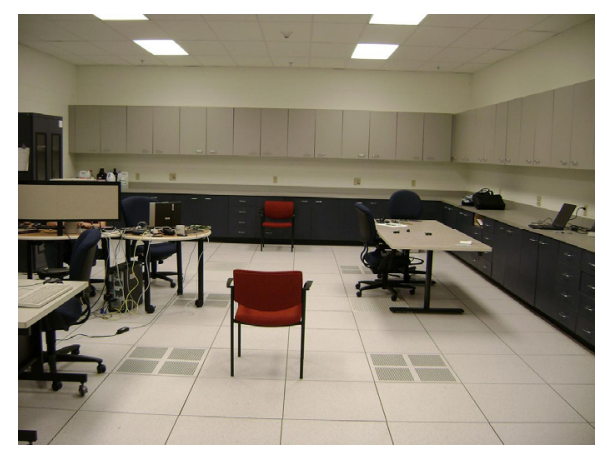

Figure 3. Indoor environment considered in our experiment. 


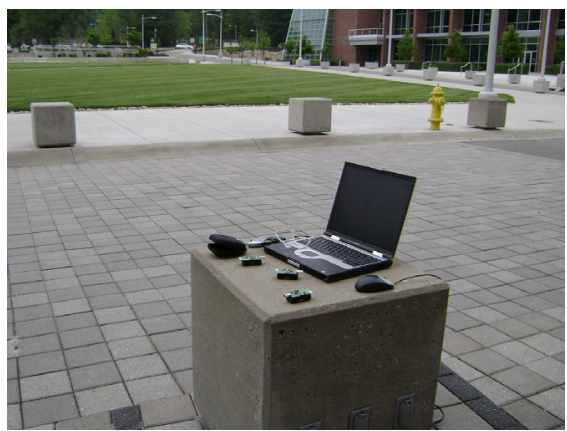

Figure 4. Outdoor environment considered in our experiment.

RSSI of the most recently received packet from the other sensor. Sensor 3 never transmits; rather, it forwards packets from sensors 1 and 2 to a workstation for analysis. The ad hoc channel between sensor 1 and 2 is time varying since both sensors move in different (variable) velocities and directions. Indoor and outdoor environments are considered. In our experiment, the indoor environment is the laboratory room shown in Figure 3, while the outdoor environment is shown in Figure 4.

In the estimation and identification process, a $4^{\text {th }}$ order ad hoc channel model as described in (10) and (11) is considered. Thus, the system parameters $\theta_{t}=\left\{A_{t}, B_{t}, C_{t}, D_{t}\right\}$ can be represented as:

$$
\begin{aligned}
& A_{t}=\left[\begin{array}{llll}
0 & 1 & 0 & 0 \\
a_{1} & a_{2} & 0 & 0 \\
0 & 0 & 0 & 1 \\
0 & 0 & a_{3} & a_{4}
\end{array}\right], B_{t}=\left[\begin{array}{llll}
b_{1} & \delta_{12} & \delta_{13} & \delta_{14} \\
b_{2} & \delta_{22} & \delta_{23} & \delta_{24} \\
\delta_{31} & \delta_{32} & b_{3} & \delta_{34} \\
\delta_{41} & \delta_{42} & b_{43} & \delta_{44}
\end{array}\right],
\end{aligned}
$$

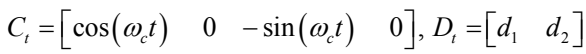

The estimation includes the channel parameters, inphase and quadrature components, and the received signal, which are then compared to the ones obtained from measurement data. It is assumed that the received signal measurement data are corrupted by white noise sequences. Figures 5 and 6 show respectively the indoor and outdoor measured and estimated received signal using the EM algorithm together with Kalman filter for 500 sampled data taken from measurements between sensor 1 and 2. The indoor system parameters are estimated as:

$$
\begin{aligned}
\hat{A} & =\left[\begin{array}{cccc}
0 & 1 & 0 & 0 \\
-0.3066 & 0.0016 & 0 & 0 \\
0 & 0 & 0 & 1 \\
0 & 0 & -0.5940 & 0.0059
\end{array}\right], \\
\hat{B}^{2} & =\left[\begin{array}{crrc}
0.8531 & -0.0369 & -0.0284 & 2.8531 * 10^{-4} \\
-0.3962 & 0.0649 & 0.0032 & -0.0193 \\
-0.0742 & 0.0074 & 0.0753 & 0.0021 \\
3.1804 * 10^{-4} & -0.0532 & 0.0064 & 0.0853
\end{array}\right], \\
\hat{C} & =\left[\begin{array}{llll}
\cos \left(\omega_{c} t\right) & 0 & -\sin \left(\omega_{c} t\right) & 0
\end{array}\right], \hat{D}^{2}=[2.0262] .
\end{aligned}
$$

while the outdoor system parameters are estimated as:

$$
\begin{aligned}
\hat{A} & =\left[\begin{array}{cccc}
0 & 1 & 0 & 0 \\
-0.7151 & 0.0037 & 0 & 0 \\
0 & 0 & 0 & 1 \\
0 & 0 & -0.1500 & 0.0515
\end{array}\right], \\
\hat{B}^{2} & =\left[\begin{array}{cccc}
0.5824 & -0.0735 & -0.0735 & 1.5395 * 10^{-4} \\
-0.7452 & 0.0846 & 0.0083 & -0.0375 \\
-0.0864 & 0.0365 & 0.0454 & 0.0264 \\
1.8643 * 10^{-4} & -0.0643 & 0.0820 & 0.0753
\end{array}\right], \\
\hat{C} & =\left[\begin{array}{llll}
\cos \left(\omega_{c} t\right) & 0 & -\sin \left(\omega_{c} t\right) & 0
\end{array}\right], \hat{D}^{2}=[1.1725] .
\end{aligned}
$$

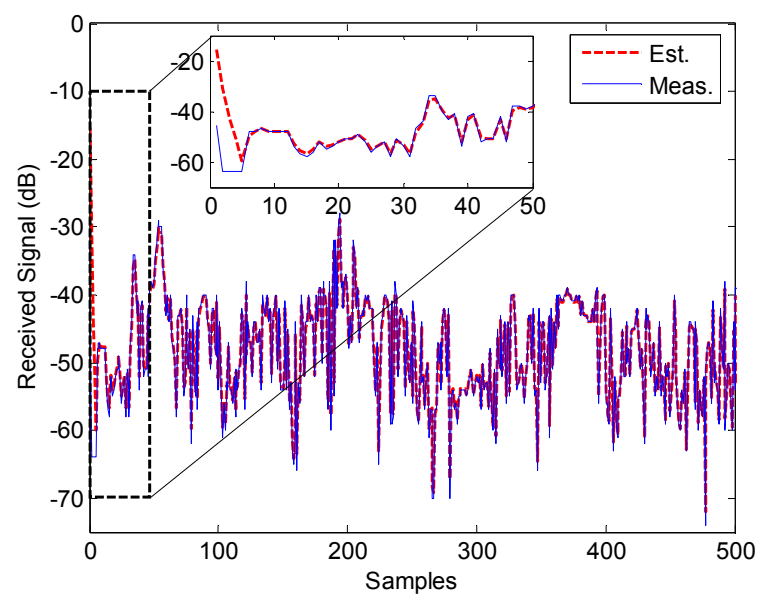

Figure 5. Indoor measured and estimated received signal from sensor 2 by using a $4^{\text {th }}$ order ad hoc channel model.

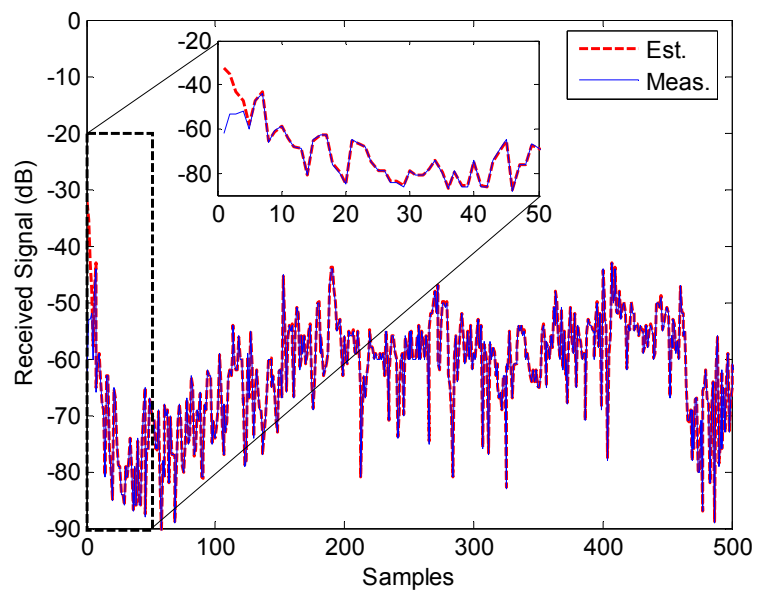

Figure 6. Outdoor measured and estimated received signal from sensor 2 by using a $4^{\text {th }}$ order ad hoc channel model. 


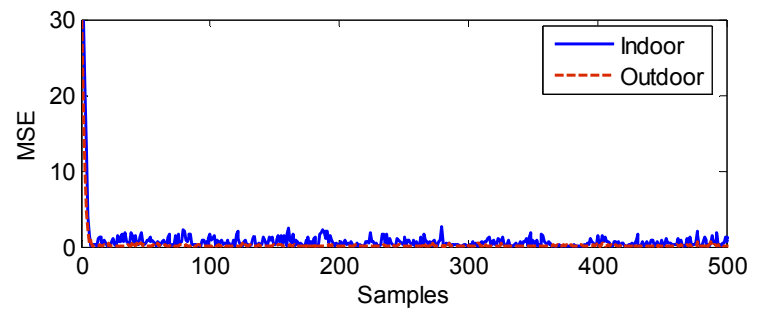

Figure 7. Received signal estimates RMSE for indoor and outdoor environments using the EM algorithm together with the Kalman filter.

From Figures 5 and 6 , it can be noticed that the received signals from indoor and outdoor wireless ad hoc channels have been estimated with very high accuracy. It takes few iterations (about 5 iterations) for the estimation algorithm to converge. The root mean square errors (RMSE) for indoor and outdoor environments are shown in Figure 7. It can be seen that indoor RMSE is higher than the one for outdoor because of reflections from walls and objects in indoor environment.

\section{CONCLUSION}

This paper describes a general scheme for extracting time varying mathematical ad hoc channel models from noisy received signal measurements. The channel models are represented in stochastic state space form. The proposed algorithm consists of filtering based on the Kalman filter to remove noise from data, and identification based on the expectation maximization (EM) algorithm to determine the parameters of the model which best describe the measurements. Indoor and outdoor experimental setups are considered. Experimental results indicate that the measured data can be generated through a simple $4^{\text {th }}$ order discrete-time stochastic differential equation.

\section{ACKNOWLEDGMENTS}

Our thanks to Prof. C.D. Charalambous for his helpful comments and discussions. The work was performed during visits of $\mathrm{M}$.
Olama, Y. Li, and T. Goodspeed at the Computational Sciences and Engineering Division, Oak Ridge National Laboratory (ORNL).

\section{REFERENCES}

[1] Olama, M.M., Djouadi, S.M., and Charalambous, C.D. 2006. Stochastic channel modeling for ad hoc wireless networks. In Proceedings of the American Control Conference (Minneapolis, Minnesota USA, June 14-16, 2006), ACC '06, $6075-6080$

[2] Charalambous, C.D., Djouadi, S.M., and Denic, S.Z. 2005. Stochastic power control for wireless networks via SDE's: Probabilistic QoS measures. IEEE Transaction on Information Theory, 51, 2 (Dec. 2005), 4396-4401.

[3] Patel, C.S., Stuber, G.L., and Pratt, T.G. 2005. Simulation of Rayleigh-faded mobile-to-mobile communication channels. IEEE Transaction on Communications, 53, 11 (Nov. 2005), 1876-1884

[4] Wang, R., and Cox, D. 2002. Double mobility mitigates fading in ad hoc wireless networks. In Proceedings of the International Symposium on Antennas and Propagation, (Kanagawa, Japan, November 26-28, 2002), 306-309.

[5] Proakis, J.G. 2000. Digital Communications, Fourth Edition, McGraw Hill, New York.

[6] Charalambous, C.D., and Logothetis, A. 2000. Maximumlikelihood parameter estimation from incomplete data via the sensitivity equations: The continuous-time case. IEEE Transaction on Automatic Control, 45, 5 (May 2000), 928934.

[7] Bishop, G., and Welch, G. 2001. An introduction to the Kalman filters, University of North Carolina.

[8] Chipcon CC2420 RF Transceiver Chip Data Sheet, Chipcon Products from Texas Instruments, DOI $=$ www.stanford.edu/class/cs244e/papers/cc2420.pdf 